\title{
Dynamic Monte-Carlo modeling of hydrogen retention and chemical erosion from Tore-Supra deposits
}

\author{
${ }^{*}$ A. Rai ${ }^{a}$, R. Schneider ${ }^{a}$, M. Warrier ${ }^{b}$, P. Roubin $^{c}$ and C. Martin ${ }^{c}$ \\ ${ }^{a}$ Max-Planck-Institut für Plasmaphysik, D-17491 Greifswald, Germany \\ ${ }^{b}$ Computational Analysis Division, BARC, Trombay, Mumbai, India - 400085 \\ ${ }^{c}$ PIIM, Université de Provence, Centre Saint-Jérôme F-13397 Marseille cedex 20, France
}

\begin{abstract}
A multi-scale model has been developed to study the hydrogen retention [1] and chemical erosion of porous graphite. To model the chemical erosion process due to thermal hydrogen ions, Küppers cycle $[2,3]$ has been introduced. The model is applied to study hydrogen transport in deposits collected from the leading edge of neutralizers of Tore Supra. The effect of internal structure on chemical erosion is studied. The MD study [4] shows that the experimentally observed decrease of erosion yield at higher fluxes is due to the decrease of carbon collision cross section at a surface due to shielding by hydrogen atom already present on the surface. Inspired by this study, a simple multi-scale model is developed to describe the flux dependence of chemical erosion. The idea is to use the local chemistry effect from the Küppers model to calculate the hydrocarbon molecule formation process and then to find the release probability of the produced hydrocarbon based on the purely geometrical constraints. The model represents quite well the trends in experimental data.
\end{abstract}

JNM keywords: Graphite, Diffusion, Monte-Carlo modeling, Plasma-material interaction PSI-18 keywords: Chemical erosion, Diffusion, Hydrogen inventory, Tore-Supra PACS codes: 52.65.Pp 
* Corresponding author e-mail: Abha.Rai@ipp.mpg.de

* Corresponding author address: HGF-NWG, Max-Planck-Institut für Plasmaphysik, Wendelsteinstrasse 1, D-17491 Greifswald, Germany.

Presenting author: Ralf Schneider

Presenting author e-mail: Ralf.Schneider@ipp.mpg.de

\section{Introduction}

Plasma material interaction in fusion devices is a critical issue that affects the overall machine performance. In the current design of ITER, graphite has been chosen as a plasma facing material (PFM). The two main topics regarding interaction of hydrogen isotopes and graphite that need to be understood properly are: (i) hydrogen retention and release and (ii) chemical erosion and its flux dependence. Tore Supra offers a unique opportunity to study the steady-state particle balance due to its ability to produce long discharges (> $200 s$ ) [5]. In long pulses, particle balance gives evidence that a constant fraction of the injected gas (typically $50 \%$ of the injected fuel) is retained in the wall for the duration of the shot, showing no sign of wall saturation after more than 6 minutes of discharge [6]. Neither implantation nor co-deposition could explain the constant retention rates observed in Tore Supra. It was speculated that the implantation of the hydrogen followed by the diffusion through the internal porosity could lead to the penetration and trapping of hydrogen much deeper than expected on the basis of penetration depth, into the graphite 
used as PFM. This gives rise to the need for a better understanding of the transport, retention and re-emission of hydrogen into the co-deposited layers and into the graphite.

In the present paper first the results for the hydrogen retention, release and chemical erosion from the Tore-Supra co-deposits have been presented. Since the present 3D kinetic Monte-Carlo (KMC) model does not reproduce the experimentally observed flux dependence of the chemical erosion another simple geometrical multi-scale model (SGMSM) has been developed. Based on SGMSM, in section 4 the results for the flux dependence of chemical erosion are presented.

\section{Structure of the Tore Supra deposits}

Based on the experimental structural analysis [7-9], we simulate the hydrogen retention and re-emission of the deposits found on the leading edge of the neutralizer (named N-LE)

of Tore Supra. The typical plasma flux near this region is about $10^{17}-10^{18} \mathrm{D}^{+} \mathrm{cm}^{-2} \mathrm{~s}^{-1}$, the tile temperature can reach up to $1500 K$ and the incident ion energy is typically 50 - $300 \mathrm{eV}$. The porosity of these deposits is multi-scale in nature consisting of micropores with typical size lower than $2 \mathrm{~nm}$ ( $\sim 11 \%$ void contribution to the total volume), mesopores (typical size between 2 and $50 \mathrm{~nm}, \sim 5 \%$ ) and macropores with a typical size more than $50 \mathrm{~nm}$. Typical void size refers to the smallest of the void size in $\mathrm{X}, \mathrm{Z}$ and $\mathrm{Y}$ directions. Transmission electron microscopy performed on thin foils cut from an ovoid reveals a regular network of parallel slit-shaped mesopore (size $\sim 10 \mathrm{~nm}$ ) and macropores 
( size $\sim 100 \mathrm{~nm}$ ), with a well-defined orientation with respect to the ovoid axis. In the present work we use the following nomenclature for different length scales:

- micro-scales: void size $<2 \mathrm{~nm}$ and system dimension of several nano-meters.

- meso-scales: void size $<10 \mathrm{~nm}$ and system dimension of several $100 \mathrm{~nm}$.

- macro-scales: void size $>50 \mathrm{~nm}$ and system dimension of several $10 \mu \mathrm{m}$.

\section{Description of 3D KMC model}

The physics of the interaction of hydrogen with graphite used in the fusion devices is multi-scale in space $(\stackrel{\AA}{A}$ to $\mathrm{cm}$ ) and time (pico-seconds to seconds). The idea is to use the insights gained from the microscopic models (MD or ab-initio methods) for modeling the transport at the meso-scale and further at the macro-scale in order to understand the physical processes contributing to macroscopic transport. The 3D multi-scale model developed by Warrier et al. [10] to simulate the "trace atom diffusion" has been improved to model the hydrogen reactive-diffusive transport (with inclusion of Küppers cycle, thermal annealing and other reactions) in porous graphite [1]. A continuous influx of hydrogen atoms determined by the flux of the ion beam has been implemented. We parametrized the recombination rate and trans-granular diffusion (TGD) coefficient for the TS deposits at meso-scales (sample having micropore and mesopores) and used them as input to model macropores at macro-scales. The statistical error of the input parameters (recombination rate and diffusion coefficient at meso-scale) is around $10 \%$ at low temperatures and reduces to $2 \%$ at higher temperatures. 


\section{1 setting up the simulation}

At meso-scales, the geometry was implemented in our simulations by creating a porous structure of $300 \mathrm{~nm}, 300 \mathrm{~nm}, 100 \mathrm{~nm}$ in $\mathrm{X}, \mathrm{Y}$ and Z directions respectively, representing a typical granule. The graphite sample was composed of micropores having $11 \%$ void fraction with void size $1 \mathrm{~nm}, 10 \mathrm{~nm}, 10 \mathrm{~nm}$ and mesopores having $5 \%$ void fraction with void size $10 \mathrm{~nm}, 18 \mathrm{~nm}, 18 \mathrm{~nm}$ in $\mathrm{X}, \mathrm{Y}$ and $\mathrm{Z}$ directions respectively. We are interested in the analysis of thermalized hydrogen. We approximate the profile of the ions (300 $e V$ ) given by TRIM calculations with Gaussian distribution with a range of penetration of $7.5 \mathrm{~nm}$ and a standard deviation of $6.0 \mathrm{~nm}$ along the $\mathrm{Z}$-direction. The deposits have $0.75 \%$ active carbon sites, therefore, in our simulation, every time an atom jumps, a trapping probability of 0.0075 is used. At macro-scales, the geometry was implemented in our simulations by creating a porous structure of $2 \mu m, 2 \mu m, 2 \mu m$ in $\mathrm{X}, \mathrm{Y}$ and Z direction respectively. Periodic boundary conditions were applied in $\mathrm{X}$ and $\mathrm{Y}$ direction. The graphite sample was composed of macropores having $10 \%$ void fraction with void size $100 \mathrm{~nm}, 100 \mathrm{~nm}, 600 \mathrm{~nm}$. For the hydrogen atom and molecule transport, KMC was used in the voids and Monte-Carlo diffusion (MCD) was used in the granules.

\subsection{Results}

Fig. 1 shows the hydrogen release behavior for mesopores and macropores. From the total released amount the fraction contributed by atoms and molecules is plotted on the vertical-axis. At meso-scales, the hydrogen release curve follows the experimental results 
of [11] and the model calculation of [12], i.e. the released flux is mainly molecular hydrogen at lower temperatures and atomic hydrogen at higher temperatures. At macro-scales, all the hydrogen atoms which are distributed on the macropore surfaces, are trapped (high trapping probability of 0.0075 ) or they diffuse deep into the bulk. The hydrogen atoms which land up in the granules diffuse within them (MCD, using TGD coefficient) and get recombined to form hydrogen molecules (determined by the recombination rate calculated at meso-scales). The atomic hydrogen which is released from the mesopores gets trapped at the macropore surfaces and the hydrogen released in the molecular form from the mesopores, due to its chemical inactivity is able to reach the geometrical surface and gets released from there. Therefore, at macro-scales even at very high temperatures hydrogen is released mainly in the molecular form.

Fig. 2 views a cut through the $3 \mathrm{D}$ geometry in the $\mathrm{X}-\mathrm{Y}$ plane located at $\mathrm{Z}=55 \mathrm{~nm}$. All the atoms having their Z-position between 50 to $60 \mathrm{~nm}$ have been projected on the $\mathrm{X}-\mathrm{Y}$ plane. It can be seen that most of the hydrogen is either adsorbed $(0.9 \mathrm{eV})$ or trapped $(2.67 \mathrm{eV})$ on the macropore surfaces. A large quantity of hydrogen is able to diffuse well beyond the implantation range $(7.5 \mathrm{~nm})$. As explained above, a lot of hydrogen released from the mesopores remains adsorbed or trapped at the macropore surfaces and a significant amount of hydrogen is present in the molecular form within the crystallites in the granules having mesopores and micropores. It gives rise to an internal inventory (or deposition) on macropore surfaces. This mechanism might play a very significant role during the chemical sputtering of such deposits. Hydrocarbons will deposit on the internal 
surfaces of the macropores. This will further enhance the tritium retention problem.

The Küppers cycle $[2,3,13]$ for interaction of hydrogen with hydrocarbons with a thermal annealing process has been included in the 3D kinetic Monte-Carlo (KMC) model. The model reproduces the temperature dependence of the erosion very well. Fig. 3 shows the point of creation of $\mathrm{H}_{2}$ and $\mathrm{CH}_{3}$ molecules in a porous structure of $10 \mathrm{~nm}, 10 \mathrm{~nm}, 6 \mathrm{~nm}$ in $\mathrm{X}, \mathrm{Y}$ and $\mathrm{Z}$ direction respectively, with periodic boundary conditions in $\mathrm{X}$ and $\mathrm{Y}$ directions. The graphite sample was composed of micropores having $12 \%$ void fraction with a cubic void of size $1 \mathrm{~nm}$. Hydrogen molecules are created within as well as much beyond the implantation zone. The formation of $\mathrm{CH}_{3}$ molecules is confined to the hydrogen implantation range as observed in experiments [14]. The hydrogen atoms diffusing along the micro-void surfaces penetrate beyond the penetration depth and on the way form molecules, after which they are quickly released. For the formation of $\mathrm{CH}_{3}$ at least 4 hydrogenation events should take place. Due to the high $\mathrm{H}-\mathrm{H}$ recombination probability at this temperature $(750 \mathrm{~K})$ very few hydrogen atoms are left for the multiple hydrogenation events and formation of $\mathrm{CH}_{3}$. That is why practically no $\mathrm{CH}_{3}$ is formed beyond the implantation region. It was also observed that the sample having higher internal surface area facilitates the chemical erosion process. This again emphasizes the fact that in order to make reasonable prediction of the erosion problem in future fusion devices, the detailed internal structure of the graphite sample should be taken into account.

The local chemistry picture of chemical erosion from the Küppers cycle is not sufficient 
to reproduce the flux dependence of erosion yield. Salonen et al. performed MD studies to understand the flux dependence of chemical erosion $[4,15]$. A a:C-H sample was bombarded with hydrogen ions of $10 \mathrm{eV}$ energy with a rate of $2 \times 10^{29}$ ions $/ \mathrm{m}^{2} \mathrm{~s}$. The hydrogen content of the surface first increased strongly, and, after reaching a $\mathrm{H} / \mathrm{C} \sim 0.56$ the sample became supersaturated. The erosion yield for the unsaturated surface was $\sim$ 0.01, while for the supersaturated surface it was only $\sim 0.001$. The drop of one order of magnitude in the observed erosion yield was due to the decreased carbon collision cross section at a surface which had obtained the temporary supersaturation of $\mathrm{H}$ atoms due to the extremely high unrealistic fluxes involved. Inspired by the above study, a simple multi-scale model was developed which can account for the flux dependence of the erosion yield of carbon.

\section{Simple geometrical multi-scale model}

The underlying philosophy is that due to the shielding of the more deep lying carbon atoms, the origin of the total amount of carbon that can be released is limited to few surface layers only. The hydrocarbon molecules which are present in the deeper layers collide with the other target atoms in the attempt to reach the surface. This geometrical constraint faced by the hydrocarbon molecule is the main reason which gives rise to the strong flux dependence. The idea is to use the local chemistry effect from the Küppers model to calculate the hydrocarbon molecule formation process and then to find the release

probability of the produced hydrocarbon based on the purely geometrical constraints. 
Chemical erosion yield obtained from the 3D KMC model, $Y_{\text {Chem }}$, gives the number of $\mathrm{CH}_{3}$ molecules produced per incident hydrogen atom from the sample with given internal structure, at a given temperature and ion energy. Then a ray tracing kind of technique was used in the $3 \mathrm{D}$ spherical volume to find $P_{\text {Geometry }}$ (release probability of the hit hydrocarbon molecule on the basis of geometrical constraints). $P_{\text {Geometry }}$ was calculated from the ratio of the number of hits with other carbon atoms (lying more shallowly than the chosen carbon atom) to the total number of trials. We start with an ordered graphite structure, and, after the hydrogen ions hit the carbon atoms, the structure of the sample is updated. The release probability calculated by this method is due to the geometry of the sample $\left(P_{\text {Geometry }}\right)$ and the location of the particular carbon atom hit by the hydrogen atom. The actual release probability $\left(P_{\text {Release }}\right)$ is

$$
P_{\text {Release }}=P_{\text {Geometry }} \times Y_{\text {Chem }}
$$

So the quantity $P_{\text {Release }}$, includes the effect of the chemistry processes proposed by Küppers

et al. [2] as well as the effect of the geometrical constraints on the carbon release probability due to the roughness of the sample and the position of the hit carbon atom.

\subsection{Setting up the simulation}

A crystalline graphite sample of $4.9 \mathrm{~nm} \times 4.3 \mathrm{~nm} \times 13.4 \mathrm{~nm}$ was created, having 32000 carbon atoms in the simulation volume. The sample was bombarded with $n_{H}$ number of hydrogen atoms per simulation time step, with $n_{H}$ ranging from 1 to $8000 . Y_{\text {Chem }}$ in the present simulations was taken to be 0.08 which is the typical erosion yield obtained 
from the $3 \mathrm{D} \mathrm{KMC}$ model. At low fluxes $\left(n_{H}=1\right)$, on average the $P_{\text {Geometry }}$ of a hit Hydrocarbon molecule close to the surface is 0.5 and this leads to $P_{\text {Release }}$ of 0.04 (flux, $\phi=10^{19} \mathrm{H} \mathrm{m}^{-2} \mathrm{~s}^{-1}$, Fig. 4). This value matches the experiments for low flux range (Fig. 3 of ref. [16]). In the experiments at fluxes $\left(<2 \times 10^{20} \mathrm{H} \mathrm{m}^{-2} \mathrm{~s}^{-1}\right)$ the erosion yield is practically constant and at higher fluxes it shows a $\phi^{-0.7}$ dependence. Since time is the only free parameter in the SGMSM, one time calibration against the experimental data is done to assign a corresponding flux value to the incident $n_{H}$ value. The case with $n_{H}$ of 5 atoms represents the flux of $10^{19} \mathrm{H} \mathrm{m}^{-2} \mathrm{~s}^{-1}$.

\subsection{Results}

Fig. 4 shows the erosion yield calculated from the simple multi-scale model and the experimental data. The erosion yield calculated from the present model matches very well with the experimental data. We propose the following mechanism for the observed flux dependence. Until incident hydrogen flux $\leq 2 \times 10^{20} \mathrm{H} \mathrm{m}^{-2} \mathrm{~s}^{-1}$, the hydrogen atomic density is low enough such that each incoming $\mathrm{H}$-atom gets access to the carbon atom and the erosion yield is mainly determined by $Y_{\text {Chem }}$ (erosion yield coming from 3D KMC model). So the released carbon flux $\Gamma_{C}$ increases practically linearly with incident hydrogen flux $\Gamma_{H}$ and the erosion yield $\mathrm{Y}\left(=\Gamma_{C} / \Gamma_{H}\right)$ remains constant. Due to the low energy of the incoming hydrogen ions $(\sim 30 \mathrm{eV})$ only few surface layers are accessible and therefore the maximum number of carbon atoms that can be released is determined by the number of carbon atoms present within these surface layers. As the flux increases further the hydro- 
gen atomic density increases close to the surface and gets a kind of supersaturation. The supersaturation of the surface layers leads to a reduced collision cross-section or shielding of the more deep lying carbon layers. Due to this reason the $\Gamma_{C}$ remains practically constant (determined by the maximum number of carbon atoms present within the few surface layers) for flux values higher than $\left(\leq 2 \times 10^{20} \mathrm{H} \mathrm{m}^{-2} \mathrm{~s}^{-1}\right)$ and therefore if we still increase $\Gamma_{H}$, erosion yield $\mathrm{Y}\left(=\Gamma_{C} / \Gamma_{H}\right)$ will obviously decrease. This is one possible mechanism responsible for the observed flux dependence.

The hydrocarbon molecule production takes place at the end of the penetration depth of the incident ions and depends on the internal structure of the carbon sample, therefore, the internal structure of the sample mainly affects $Y_{\text {Chem }}$ slightly. Whereas the release probability is mainly determined by $P_{\text {Geometry }}$ which depends very strongly on the incident ion flux rather than the internal structure of the sample. So the present flux dependence showed practically no dependence on the internal structure of the sample. The results presented above shows that the flux dependence of erosion yield is indeed very strong. This means that the problem of carbon erosion and co-deposition will not be as severe as predicted and carbon might still be a good candidate for a PFM for ITER. 


\section{$5 \quad$ Summary}

The simulations show that in case of multi-scale porosity, the macropores play the dominant role in retention and release behavior of hydrogen. Hydrogen released from the micropores and mesopores gets adsorbed on the surfaces of the macropores. This kind of internal deposition of hydrogen is very serious and will further hinder tritium recovery from co-deposits. The higher internal surface area of the sample facilitates the molecule formation process. The simple geometrical multi-scale model, using the input from the local chemistry picture of chemical erosion gives the experimentally observed flux depen-

dence. The result shows that if the efficient ways of tritium recovery (from the internal depositions) are used then carbon might still be a good candidate for a PFM for ITER.

\section{Acknowledgments}

R. Schneider and A. Rai acknowledge funding of the work by the Initiative and Networking Fund of the Helmholtz Association. M. Warrier acknowledges funding from the MaxPlanck India Fellowship.

\section{References}

[1] A. Rai, R. Schneider, and M. Warrier. J. Nucl. Mater, 374:304-312, (2008).

[2] J. Küppers. Surface Science Reports, 22:249-321, (1995). 
[3] M. Wittmann and J. Küppers. J. Nucl. Mater., 227:186-194, (1996).

[4] E. Salonen et al. J. Nucl. Mater., 290-293:144-147, (2001).

[5] B. Pégourié et al. Physics Scripta, T111:23-28, (2004).

[6] E. Tsitrone et al. J. Nucl. Mater., 337-339:539-543, (2005).

[7] C. Martin et al. J. Nucl. Mater., 363-365:1251-1255, (2007).

[8] P. Roubin et al. J. Nucl. Mater., 337-339:990-994, (2005).

[9] M. Richou et al. Carbon, 45(14):2723-2731, (2007).

[10] M. Warrier. Ph.D. Dissertation, Faculty of Mathematics and Natural Science, Uni. Greifswald, Germany, (2004).

[11] P. Franzen and E. Vietzke. J. Vac. Sci. Technol. A, 12:820-825, (1994).

[12] A. A. Haasz et al. J. Appl. Phys., 77(1):66-86, (1995).

[13] A. A. Haasz B. V. Mech and J. W. Davis. J. Appl. Phys., 84(3):1655-1669, (1998).

[14] W. Möller and B. M. U. Scherzer. Appl. Phys. Lett., 50(26):1870-1872, (1987).

[15] E. Salonen et al. Phys. Rev. B, 60:14005-14008, (1999).

[16] J. Roth et al. Nucl. Fus., 44(11):L21-L25, (2004).

[17] M. Balden and J. Roth. J. Nucl. Mater., 280:39, (2000).

[18] R. Pugno et al. Last results from the hydrocarbon puff experiment in the ASDEX upgrade divertor, EU Task Force on PWI Meeting, 2004. Garching, February, http://www.efdataskforce-pwi.org/. 


\section{List of Figures}

1 Released amount of hydrogen atoms and molecules as a function of temperature for mesopores and macropores. Hydrogen atoms and molecules for mesopores are represented by curve 1 and 2 respectively. Similarly, for macropores hydrogen atoms and molecules are represented by curve 3 and 4 respectively.

2 A view of the $\mathrm{X}-\mathrm{Y}$ plane located at $\mathrm{Z}=55 \mathrm{~nm}$. The white, grey and pale colored regions correspond to void, surface and the bulk of the graphite respectively. Open green circle: adsorbed hydrogen atom, red dots: trapped hydrogen atom, cyan dots: $H_{2}$ molecules in the crystallites, magenta: $H_{2}$ molecules in the void and blue dots: two trapped hydrogen atoms close enough to form a molecule.

3 Depth profile showing the point of origin of $\mathrm{H}_{2}$ and $\mathrm{CH}_{3}$ molecules. $\mathrm{H}_{\text {initial }}$ shows the initial profile of the incident hydrogen atoms.

4 Chemical erosion yield as a function of the incident flux at room temperature. The result from the present work is compared with the data from ion beam experiments [17] and the divertor of ASDEX Upgrade [18] (All data are published in [16]). The solid line is a fit to the experimental data $[16]$. 


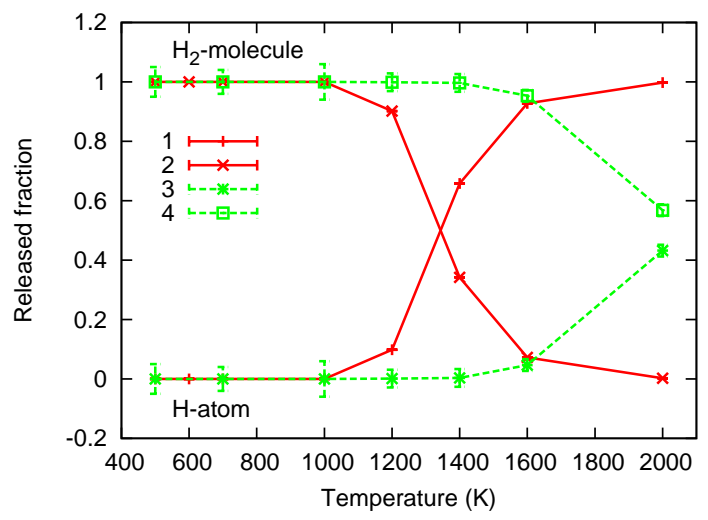

Fig. 1. Released amount of hydrogen atoms and molecules as a function of temperature for mesopores and macropores. Hydrogen atoms and molecules for mesopores are represented by curve 1 and 2 respectively. Similarly, for macropores hydrogen atoms and molecules are represented by curve 3 and 4 respectively. 


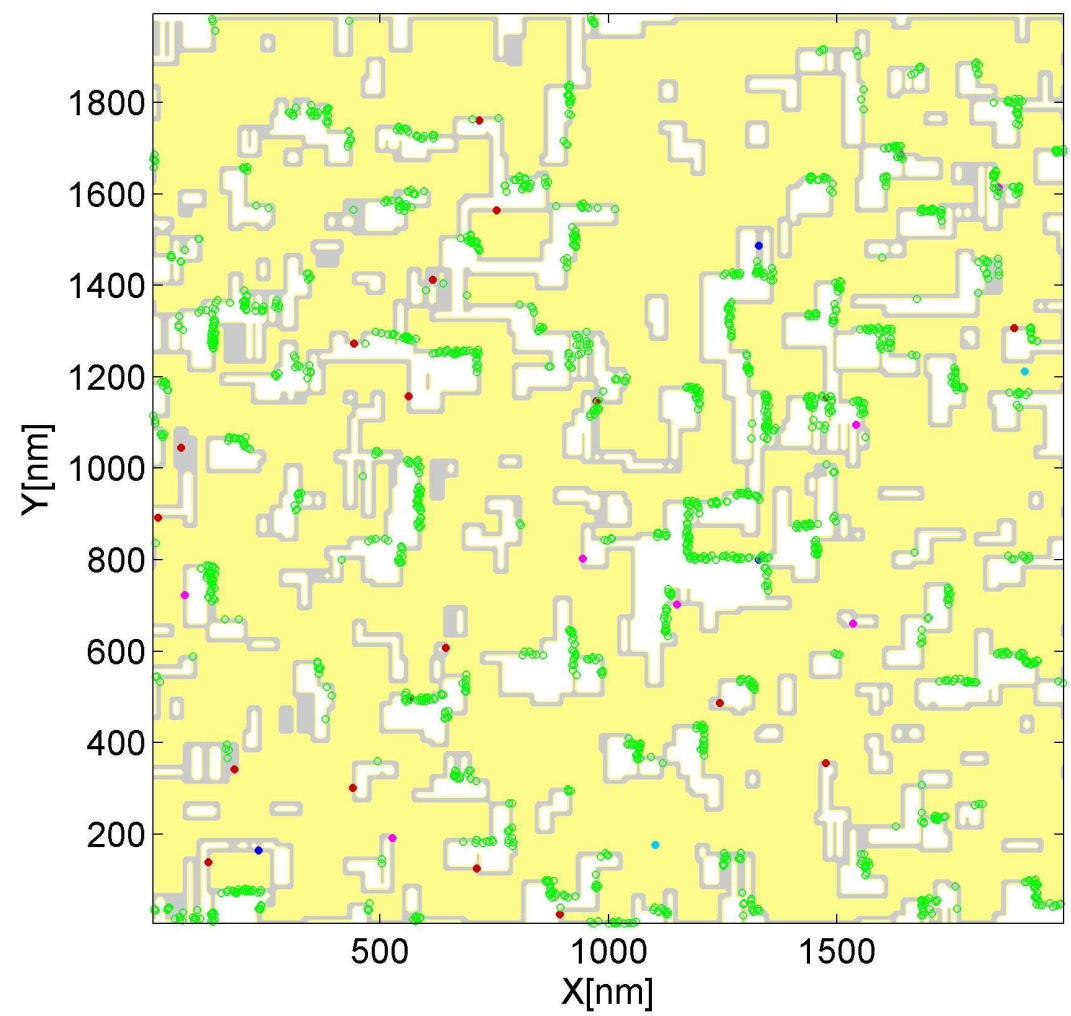

Fig. 2. A view of the $\mathrm{X}-\mathrm{Y}$ plane located at $\mathrm{Z}=55 \mathrm{~nm}$. The white, grey and pale colored regions correspond to void, surface and the bulk of the graphite respectively. Open green circle: adsorbed hydrogen atom, red dots: trapped hydrogen atom, cyan dots: $H_{2}$ molecules in the crystallites, magenta: $\mathrm{H}_{2}$ molecules in the void and blue dots: two trapped hydrogen atoms close enough to form a molecule. 


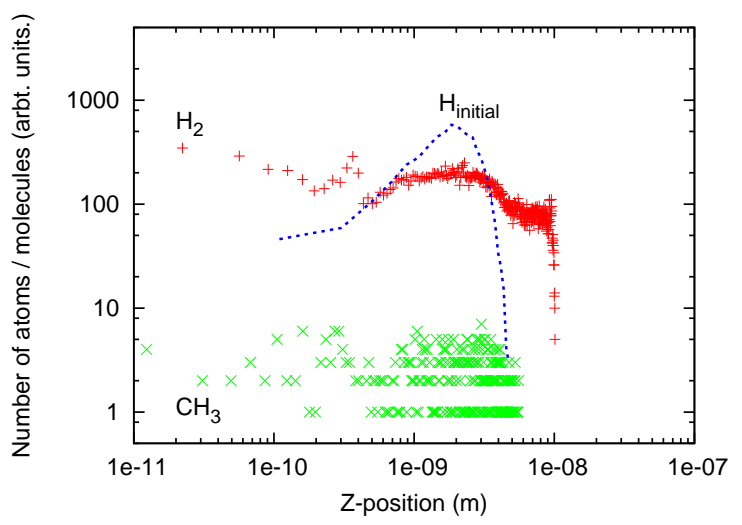

Fig. 3. Depth profile showing the point of origin of $\mathrm{H}_{2}$ and $\mathrm{CH}_{3}$ molecules. $\mathrm{H}_{\text {initial }}$ shows the initial profile of the incident hydrogen atoms. 


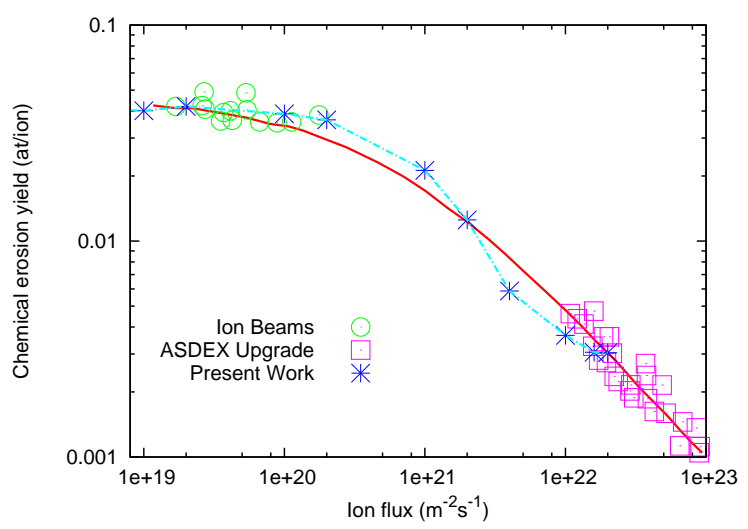

Fig. 4. Chemical erosion yield as a function of the incident flux at room temperature. The result from the present work is compared with the data from ion beam experiments [17] and the divertor of ASDEX Upgrade [18] (All data are published in [16]). The solid line is a fit to the experimental data [16]. 